\title{
Characterization of laser-driven Radiative shock of Astrophysical interests
}

\author{
U. Chaulagain ${ }^{1 \dagger}$, C. Stehlé ${ }^{1}$, J. Larour ${ }^{2}$, M. Kozlová ${ }^{3}$, \\ F. Suzuki-Vidal ${ }^{4}$, J. Nejdl ${ }^{3}$ and R. L. Singh ${ }^{1,2}$
}

${ }^{1}$ LERMA, Observatoire de Paris, UPMC, CNRS, 5 Pl. Janssen, 92195 Meudon, France, ${ }^{2}$ LPP, Ecole Polytechnique, UPMC, CNRS, 91128 Palaiseau, France, ${ }^{3}$ Institute of Physics of ASCR, Na Slovance 2, 18221 Prague 8, Czech Republic, ${ }^{4}$ The Blackett Laboratory, Imperial College,

Prince Consort Road, London SW7 2AZ, UK, ${ }^{\dagger}$ Currently at ELI Beamlines, Institute of Physics ASCR, Na Slovance 1999/2, Prague, 182 21, Czech Republic

\begin{abstract}
We highlight the recent experimental results on laser-driven radiative shock waves of astrophysical interests using kJ PALS laser facility. The generated shock is probed instantaneously by X-ray laser $(\lambda=21.2 \mathrm{~nm})$ showing an unambiguous shock structure that includes both the post-shock and the precursor.
\end{abstract}

Keywords. Radiative shock, X-ray laser, stellar accretion, laboratory astrophysics, plasma.

Radiative shocks are ubiquitous in many astrophysical systems, including stellar accretion shocks, supernovae remnants, jet driven shocks etc. In the case of stellar accretion, matter is channeled from the surrounding disk into accretion columns by the strong stellar magnetic field and falls onto the stellar photosphere at several hundred $\mathrm{km} / \mathrm{s}$. This generates a strong radiative shock with X-ray spectral signatures that are a key ingredient to quantify the mass accretion rate. Radiative shock plays a vital role in radiation hydrodynamics study as it combines both radiation and hydrodynamics in a nontrivial way (Mihalas and Mihalas (1999)). Thus understanding the physical structure and dynamics of such complex plasma provides a key knowledge of radiation hydrodynamics. With the advancement of high-energy laser and pinch facilities, it is now possible to generate a strong radiative shock in high energy density laboratory experiments. Despite the huge scale difference, both in time and space, the laboratory experiments are able to study these astrophysical processes. In addition, experiments are particularly useful to benchmark the new astrophysical radiation-hydrodynamic codes.

Recently, we have performed radiative shock experiments on the kJ scale PALS facility. The shock was generated in a xenon filled miniature cell at a fraction of bar pressure. The experimental details is presented elsewhere (Chaulagain et al. (2013)). Different set of plasma diagnostics were used to characterize the generated shock wave. The instantaneous imaging at $21.2 \mathrm{~nm}$ shows, for the first time, a complete structure of radiative shock by the presence of both the radiative precursor and the post shock (see Chaulagain et al. (2015)). The experimental results are in a good agreement with 2D radiative-hydrodynamics simulations results (Cotelo et al. (2014)).

\section{References}

Mihalas, D. \& Mihalas, B. W. 1999, Dover Pub. Inc., Mineola, New York

Chaulagain, U., Stehlé, C., Larour, J., et al. 2013, Proc. SFAA, 213-217

Chaulagain, U., Stehlé, C., Larour, J., et al. 2015, High Energy Density Physics, 17, 106-113

Cotelo, M., Velarde, P., de la Varga, A. G., et al. 2015, High Energy Density Physics, 17, 68-73 